\title{
Multiple photodetachment of silicon anions via $K$-shell excitation and ionization
}

\author{
A. Perry-Sassmannshausen $\odot,{ }^{1}$ T. Buhr $\odot,{ }^{1}$ M. Martins $\odot,{ }^{2}$ S. Reinwardt, ${ }^{2}$ F. Trinter $\odot,{ }^{3,4}$ \\ A. Müller $\odot,{ }^{5}$ S. Fritzsche $\odot,{ }^{6,7}$ and S. Schippers $\oplus^{1, *}$ \\ ${ }^{1}$ I. Physikalisches Institut, Justus-Liebig-Universität Gießen, 35392 Giessen, Germany \\ ${ }^{2}$ Institut für Experimentalphysik, Universität Hamburg, 22761 Hamburg, Germany \\ ${ }^{3}$ Institut für Kernphysik, Goethe-Universität Frankfurt am Main, 60438 Frankfurt am Main, Germany \\ ${ }^{4}$ Molecular Physics, Fritz-Haber-Institut der Max-Planck-Gesellschaft, 14195 Berlin, Germany \\ ${ }^{5}$ Institut für Atom- und Molekülphysik, Justus-Liebig-Universität Gießen, 35392 Giessen, Germany \\ ${ }^{6}$ Helmholtz-Institut Jena, 07743 Jena, Germany \\ ${ }^{7}$ Theoretisch-Physikalisches Institut, Friedrich-Schiller-Universität Jena, 07743 Jena, Germany
}

(Received 29 July 2021; accepted 1 November 2021; published 15 November 2021)

\begin{abstract}
Experimental cross sections for $m$-fold photodetachment $(m=3-6)$ of silicon anions via $K$-shell excitation and ionization were measured in the photon-energy range of $1830-1900 \mathrm{eV}$ using the photon-ion merged-beams technique at a synchrotron light source. All cross sections exhibit a threshold behavior that is masked by prethreshold resonances associated with the excitation of a $1 s$ electron to higher, either partly occupied or unoccupied atomic subshells. Results from multiconfiguration Dirac-Fock calculations agree with the experimentally derived cross sections for photoabsorption if small energy shifts are applied to the calculated resonance positions and detachment thresholds. Moreover, a systematic approach is applied for modeling the deexcitation cascades that set in after the initial creation of a $K$-shell hole. The resulting product charge-state distributions compare well with the measured ones for direct $K$-shell detachment but less well for resonant $K$-shell excitation. The present results are potentially useful for identifying silicon anions in cold plasmas such as interstellar gas clouds.
\end{abstract}

DOI: 10.1103/PhysRevA.104.053107

\section{INTRODUCTION}

Atomic anions are prototypal quantum systems for studying correlation effects since the extra electron in theses systems is bound by short-range forces. A fundamental process that enables one to probe correlation is photodetachment of atomic anions, i.e., the removal of one or more electrons by absorption of a single photon. Thorough investigations of this process have repeatedly lead to a fruitful interplay between experiment and quantum theory, in particular, for outer-shell photodetachment (see, e.g., Refs. [1,2] and references therein).

The correlation between core and valence electrons can be probed by inner-shell detachment where the valence electrons are subject to strong many-electron relaxation effects following the creation of core holes [3,4]. These effects are strongest in the case of $K$-shell detachment, which can experimentally be investigated by the photon-ion merged-beams technique using soft $\mathrm{x}$-ray beams from synchrotron light sources [5-7]. So far, $K$-inner-shell photodetachment has been studied for a number of light ions comprising $\mathrm{Li}^{-}[8,9]$, $\mathrm{B}^{-}$[10], $\mathrm{C}^{-}$[11-13], $\mathrm{O}^{-}[14,15]$, and $\mathrm{F}^{-}$[16].

Here we extend these studies to an atomic anion where the outer shell is the $M$ shell. We present experimental cross sections $\sigma_{m}$ for $m$-fold photodetachment of $\mathrm{Si}^{-}$ions with $m=$ $3,4,5,6$ resulting in the production of multiply positively

\footnotetext{
*stefan.schippers@physik.uni-giessen.de
}

charged $\mathrm{Si}^{(m-1)+}$ ions. This process can be represented as

$$
h v+\mathrm{Si}^{-} \rightarrow \mathrm{Si}^{(m-1)+}+m e^{-} .
$$

The experimental photon-energy range of $1830-1900 \mathrm{eV}$ comprises the threshold for direct photodetachment of a $K$ shell electron. Furthermore, we present theoretical results for resonant and nonresonant $\mathrm{Si}^{-}$photoabsorption. Using a recently developed theoretical toolbox [17,18], we followed in addition the complex deexcitation cascade that sets in after the initial $K$-hole creation and gives rise to photon-energydependent distributions of product-ion charge states.

Apart from addressing fundamental questions in manybody physics, the present experimental and theoretical investigations are also useful for astrophysics considering the fact that negative ions have been identified, e.g., in the interstellar medium [19]. In particular, they may help to improve the current astrophysical models for the $\mathrm{x}$-ray absorption by silicon ions [20].

\section{EXPERIMENT}

The measurements were carried out using the photon-ion merged-beams technique [21] at the PIPE facility (Photon-Ion Spectrometer at PETRA III) [22,23], which is a permanently installed end station at the photon beamline P04 [24] of the synchrotron radiation source PETRA III operated by DESY in Hamburg, Germany. Silicon anions were produced by a Cs-sputter ion source [25] with a silicon single crystal as a sputter target and a sputter potential of about $2 \mathrm{kV}^{-\mathrm{Si}^{-}}$is 
known to posses long-lived metastable levels with excitation energies of $1.36 \mathrm{eV}$ and $0.86 \mathrm{eV}$ and lifetimes of $22 \mathrm{~s}$ and more than $5.7 \mathrm{~h}$, respectively [26]. However, $\mathrm{Si}^{-}$ions produced by a Cs-sputter ion source in an earlier experiment were found to be exclusively in their $1 s^{2} 2 s^{2} 2 p^{6} 3 s^{2} 3 p^{3}{ }^{4} S_{3 / 2}$ ground level [27]. We assume that the same holds for the present experiment. After acceleration to a kinetic energy of 6 $\mathrm{keV}$, the ions were passed through an analyzing dipole magnet which was adjusted such that ${ }^{28} \mathrm{Si}^{-}$ions were selected for further transport to the photon-ion merged-beams interaction region.

In the interaction region, where the residual-gas pressure was in the mid- $10^{-10}$ mbar range, the ion beam was coaxially merged with the counterpropagating soft $\mathrm{x}$-ray photon beam over a distance of about $1.7 \mathrm{~m}$. Si ${ }^{(m-1)+}$ ions resulting from multiple photodetachment [Eq. (1)] were separated from the primary ion beam by a second dipole magnet. Inside this magnet, a Faraday cup collected the primary ion beam, while the charge-selected product ions were directed to the detector chamber. Along their flight path, they first passed through a spherical $180^{\circ}$ out-of-plane deflector to suppress background from stray electrons, photons, and ions and then entered a single-particle detector with nearly $100 \%$ detection efficiency [28].

Relative cross sections for $m$-fold photodetachment were obtained by normalizing the count rates of $\mathrm{Si}^{(m-1)+}$ product ions on the primary $\mathrm{Si}^{-}$ion current and on the photon flux measured with a calibrated photodiode. The ion current in the interaction region was up to $40 \mathrm{nA}$, and the photon flux amounted to $\sim 1.3 \times 10^{13} \mathrm{~s}^{-1}$ at a photon energy spread of about $1.75 \mathrm{eV}$. The systematic uncertainty of the measured cross sections is estimated to be $\pm 15 \%$ at $90 \%$ confidence level [22].

The photon-energy scale was calibrated by absorption measurements at the $2 p_{1 / 2}$ ionization threshold of krypton in a gas-phase electron spectrometer by using the corresponding threshold energy of $1729.5 \mathrm{eV}$ as measured by Kato et al. [29] as a reference. The remaining uncertainty of the present photon-energy scale is estimated to be $\pm 1 \mathrm{eV}$. A detailed description of the general calibration procedure has been provided recently [30,31].

The experimental photon-energy bandwidth could be adjusted by choosing the exit-slit width of the photon beamline's monochromator which houses gratings with rulings of 400 lines $/ \mathrm{mm}$ and 1200 lines $/ \mathrm{mm}$. We used a 1200 lines $/ \mathrm{mm}$ grating and exit-slit widths of $1000 \mu \mathrm{m}$ and $50 \mu \mathrm{m}$ for lowresolution and high-resolution measurements, respectively.

\section{COMPUTATIONS}

To model the photodetachment of negative ions, such as $\mathrm{Si}^{-}$, detailed computations of their level structure need to be combined with an efficient treatment of the subsequent relaxation in order to account for the stepwise autoionization and photon emission of the ions. This stepwise relaxation can be formally described by an atomic cascade that includes ions of the same element but in different charge states that are connected to each other by different (decay) processes. In this work, all calculations presented below have been performed by means of the JAC toolbox, the Jena Atomic Calculator [17], which supports the (relativistic) computation of atomic structures and processes. This toolbox has now been expanded recently to follow rather long ionization pathways. Here we shall recall just a few details about such cascade computations, while the numerical results will be discussed together with the measurements in Sec. IV.

Atomic cascades often require an enormous effort to generate, combine, and simulate all data as needed for modeling a given experiment. To analyze such cascades more systematically, JAC has recently been expanded for modeling different cascade schemes, i.e., relaxation scenarios, including the production of (inner-shell) excited atoms by photoexcitation, photoionization, or other mechanisms and the stepwise decay of the excited states via photon or electron emission [18]. Any of these schemes starts from setting up a cascade tree, i.e., the list of possibly contributing configurations and from selecting all relevant (decay) steps of the cascade.

In a cascade decay, each ion of a given ensemble therefore follows an individual relaxation pathway. Obviously, a (very) large number of such pathways occurs in the deexcitation of deep inner-shell holes owing to the different decay processes that are possible and that have to be combined. For the stepwise decay of the $K$-shell ionized $\mathrm{Si}^{-}$ion, for example, we started from the $1 s 2 s^{2} 2 p^{6} 3 s^{2} 3 p^{3}$ configuration and considered all autoionization and photon emission processes to energetically lower-lying configurations, until up to five electrons are released from the ion. For the configuration above, this results in a total of 87 configurations and about 160,000 fine-structure transitions of the $\mathrm{Si}^{q+}(q=0, \ldots, 5)$ atoms/ions that need to be taken into account.

To formalize the competition of the various Auger and photon emission processes, we distinguish within the JAC toolbox between (so-called) cascade computations and simulations [18]. While the cascade computations are performed with the goal to calculate and compile the associated finestructure Auger and photon-emission rates, the simulations then make use of these (precompiled) data in order to derive the ion distributions or any other information that may be needed. This distinction has been found helpful to deal with the quite different experimental setups and observations, e.g., photon, electron, or ion distributions, that often can be traced back to the same (single-step) processes.

Not much needs to be said about the detailed implementation of the cascade computations and simulations which has been summarized elsewhere [18,32]. The major computational effort is spent on the representation of the fine-structure levels at each step of the cascade as well as on the calculation of the transition amplitudes. Within JAC, these are the building blocks that enable us to formulate (and implement) all cascade computations. Because of the complexity of atomic cascades, the (quantum-mechanical) representation of the fine-structure levels usually needs to be simplified by tuning the amount of the interelectronic interactions accounted for in the calculations. In the JAC toolbox, this need for simplification is captured by an hierarchy of cascade approaches for representing all the ionic level energies and state functions. For $\mathrm{Si}^{-}$, unfortunately, we were able to apply only the simplest of these approaches, the so-called averaged single-configuration approach, in which all fine-structure states are approximated by single-configuration state functions and by just the orbitals 


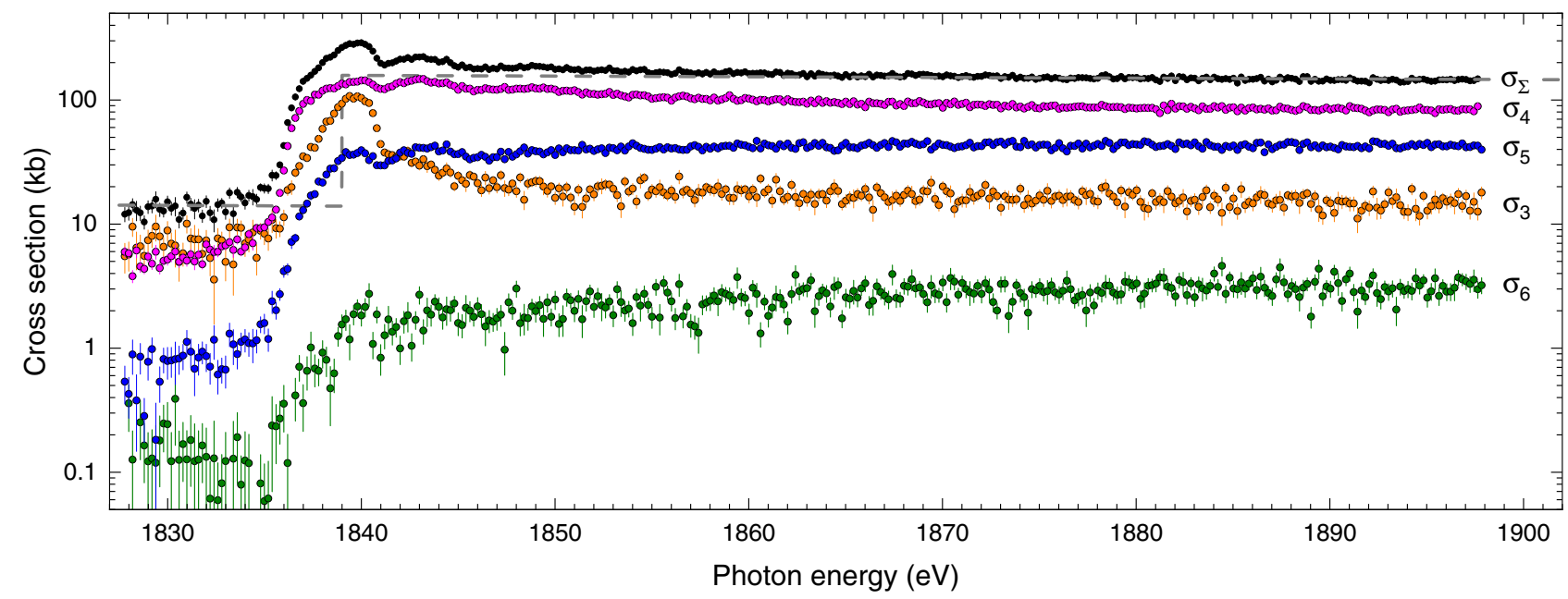

FIG. 1. Overview of the measured cross sections $\sigma_{m}$ for multiple [ $m$-fold, Eq. (1)] detachment of $\mathrm{Si}^{-}$ions. The differently colored data points represent $\sigma_{m}$ for $m=3$ (orange), $m=4$ (magenta), $m=5$ (blue), and $m=6$ (green). The error bars only account for the statistical experimental uncertainties. The black circles represent the sum $\sigma_{\Sigma}$ [Eq. (2)] of all measured cross sections $\sigma_{m}$. The dashed gray curve is the cross section for photoabsorption of neutral silicon atoms from the compilation of Henke et al. [34]. The width of the monochromator exit slit was $1000 \mu \mathrm{m}$. The distorted resonance line shapes are due to a non-Gaussian contribution to the photon-energy distribution, which becomes noticeable at large exit-slit widths for photon energies well above $1000 \mathrm{eV}$.

from the initial configuration of the cascades. This approach neglects all configuration mixing between the bound-state levels and restricts the computations to just the Coulomb interaction among the electrons as well as a single set of continuum orbitals for each step of the cascade [33].

Cascade computations have been performed for the photoabsorption and photoionization cross sections in the region of the $1 s \rightarrow 3 p, 4 p$ inner-shell excitations and $1 s$ ionization. In general, the size of such cascade computations increases rapidly with (i) the number of electrons, that need to be replaced, and (ii) the depth of the cascade, i.e., the maximum number of electrons that can be released and, as outlined above, by the number of fine-structure transitions. This increase also results in an approximate treatment of the fine-structure representation of the ionic levels that is likely the main reason for the discrepancies of the predicted ion distributions, when compared with the measurements.

\section{RESULTS AND DISCUSSION}

The measured low-resolution cross sections for multiple detachment of $\mathrm{Si}^{-}$are displayed in Fig. 1. All cross sections exhibit a rise at a photon energy of about $1835 \mathrm{eV}$ which is associated with the resonant excitation of a $1 s$ electron to higher subshells. Two rather broad resonance features at $\sim 1840$ and $\sim 1843 \mathrm{eV}$ can be discerned in all four cross-section curves. At higher energies, where the cross sections are dominated by nonresonant direct removal of a $K$-shell electron, they are structureless. The corresponding detachment thresholds are masked by the below-threshold resonances.

The $K$-shell hole, that is created either by resonant excitation or by nonresonant detachment, is filled by a cascade of radiative and Auger processes, which results in the observed energy-dependent distributions over the various product charge states analogous to what we have observed previously for $\mathrm{C}^{-}$[13], where $\mathrm{C}^{(m-1)+}$ product charge states were detected for $2 \leqslant m \leqslant 6$. As compared to the $\mathrm{C}^{-}$cross sections, the present $\mathrm{Si}^{-}$cross sections are almost an order of magnitude smaller. This follows the general trend of decreasing photoionization cross sections as the nuclear charge increases. Another significant difference between $\mathrm{C}^{-}$and $\mathrm{Si}^{-}$ concerns the dominant detachment channel which is $\sigma_{2}$ for $\mathrm{C}^{-}$ and $\sigma_{4}$ for $\mathrm{Si}^{-}$. This can be attributed to the higher number of electrons outside the $K$ shell in the $\mathrm{Si}^{-}$ion which supports many more deexcitation channels as compared to $\mathrm{C}^{-}$. In particular, the number of possibilities for autoionization increases when going from $\mathrm{C}^{-}$to $\mathrm{Si}^{-}$.

The measured relative cross sections were put on an absolute scale by matching the sum cross section

$$
\sigma_{\Sigma}=\sum_{m=3}^{6} \sigma_{m}
$$

with the absorption cross section of neutral silicon atoms [34] which is also displayed in Fig. 1. Apparently, this cross section represents the energy dependence of the experimental cross section very well except for the energies where resonances occur. In order to bring $\sigma_{\Sigma}$ to the same scale, all cross sections $\sigma_{m}$ were multiplied by the same appropriate factor. As shown explicitly for $\mathrm{C}^{-}$[7], where independently absolute cross sections were experimentally determined [13], this procedure is valid if one can safely assume that $\sigma_{\Sigma}$ represents all significant absorption channels. This assumption appears justified since the behavior of the cross sections $\sigma_{m}$ suggests that the unmeasured single and double detachment channels and the higher detachment channels with $m>6$ are negligible. The single-detachment channel could not be measured since our experimental setup is only capable of measuring charged product ions. Moreover, the measurement of the (weak) double-detachment channel was severely hampered by a large background from collisions of $\mathrm{Si}^{-}$with the residual gas such that meaningful results could not be obtained within 
a reasonable amount of time. Measurements of the multipledetachment cross sections for $m>6$ were not attempted.

The nonzero cross sections below $1835 \mathrm{eV}$ are due to direct detachment of an $L$-shell electron and the subsequent (multiple) autoionization. In this energy range, the sum cross section $\sigma_{\Sigma}$ agrees, as mentioned above, well with the cross section for photoabsorption of neutral silicon atoms. This is also the case for energies above $1855 \mathrm{eV}$ which are beyond the threshold for the direct detachment of a $K$-shell electron. For neutral silicon this threshold has been predicted to be at $1838.9 \mathrm{eV}$ [34] (dashed line in Fig. 1). For $\mathrm{Si}^{-}$, the corresponding steplike cross-section features are masked by the resonances that occur immediately below these thresholds.

In order to obtain more detailed experimental information on the photodetachment resonances we performed additional cross-section measurements with higher resolution as compared to the measurements shown in Fig. 1. These high-resolution measurements are displayed in Fig. 2. The statistical uncertainties of $\sigma_{3}$ are comparatively large because a substantial background contributed to the measured signal in this channel. Such a background was largely absent in the other scrutinized channels. In all measured cross sections, the strongest feature is a comparatively narrow resonance at $\sim 1838.4 \mathrm{eV}$ with an experimental width of $0.55 \mathrm{eV}$. A second, broader resonance feature at about $1842.4 \mathrm{eV}$ with a width of $2-3 \mathrm{eV}$ is visible in the cross sections for fourfold, fivefold, and sixfold detachment. The relative strength of this feature, when compared to the narrower one, increases with increasing product-ion charge state.

A double-peak structure was similarly observed in the multiple photodetachment of $\mathrm{C}^{-}$[13], where the first resonance was attributed to a $1 s \rightarrow 2 p$ excitation to the $1 s 2 s^{2} 2 p^{4}{ }^{4} P$ term and the second, broader resonance peak was attributed to higher excitations to ${ }^{4} P$ terms mainly of the $1 s 2 s^{2} 2 p^{3} 3 p$ configuration. In analogy, one might therefore assign the $1 s^{-1} 3 p^{4}{ }^{4} P$ term to the first $K$-shell excited resonance of $\mathrm{Si}^{-}$ and a blend of several ${ }^{4} P$ terms associated with $1 s \rightarrow 4 p$ excitations to the second resonance peak. Our calculations confirm this expectation, though one should keep in mind that they only provide a coarse orientation on the positions. For any negative ion, the calculated levels result from strong configuration interactions.

Figure 3 shows the comparison between the sum of the experimental cross sections from Fig. 2 and our theoretical absorption cross section. The individual theoretical resonances are represented by Voigt line profiles with a Gaussian width of $0.05 \mathrm{eV}$ that accounts for the experimental photon-energy spread. The calculated Lorentzian width of virtually all these resonances amounts to $0.57 \mathrm{eV}$. It is determined mainly by the KLL Auger transition that fills the core hole and it is about $15 \%$ larger than the $K$-level width of neutral silicon [35]. An energy shift of $-1.3 \mathrm{eV}$ was applied to the theoretical resonance positions in order to line these up with the experimental peak positions. This shift is within the uncertainty of the theoretical resonance energies. In order to achieve the best possible agreement between theory and experiment, a shift of similar magnitude was applied to the calculated threshold energies for direct $K$-shell detachment resulting in the values 1842.5 and $1843.3 \mathrm{eV}$ for the $1 s^{-1} 3 p^{3}\left({ }^{4} S\right){ }^{5} S_{2}$ and $1 s^{-1} 3 p^{3}\left({ }^{4} S\right)^{3} S_{1}$ levels in neutral silicon, respectively. Apart

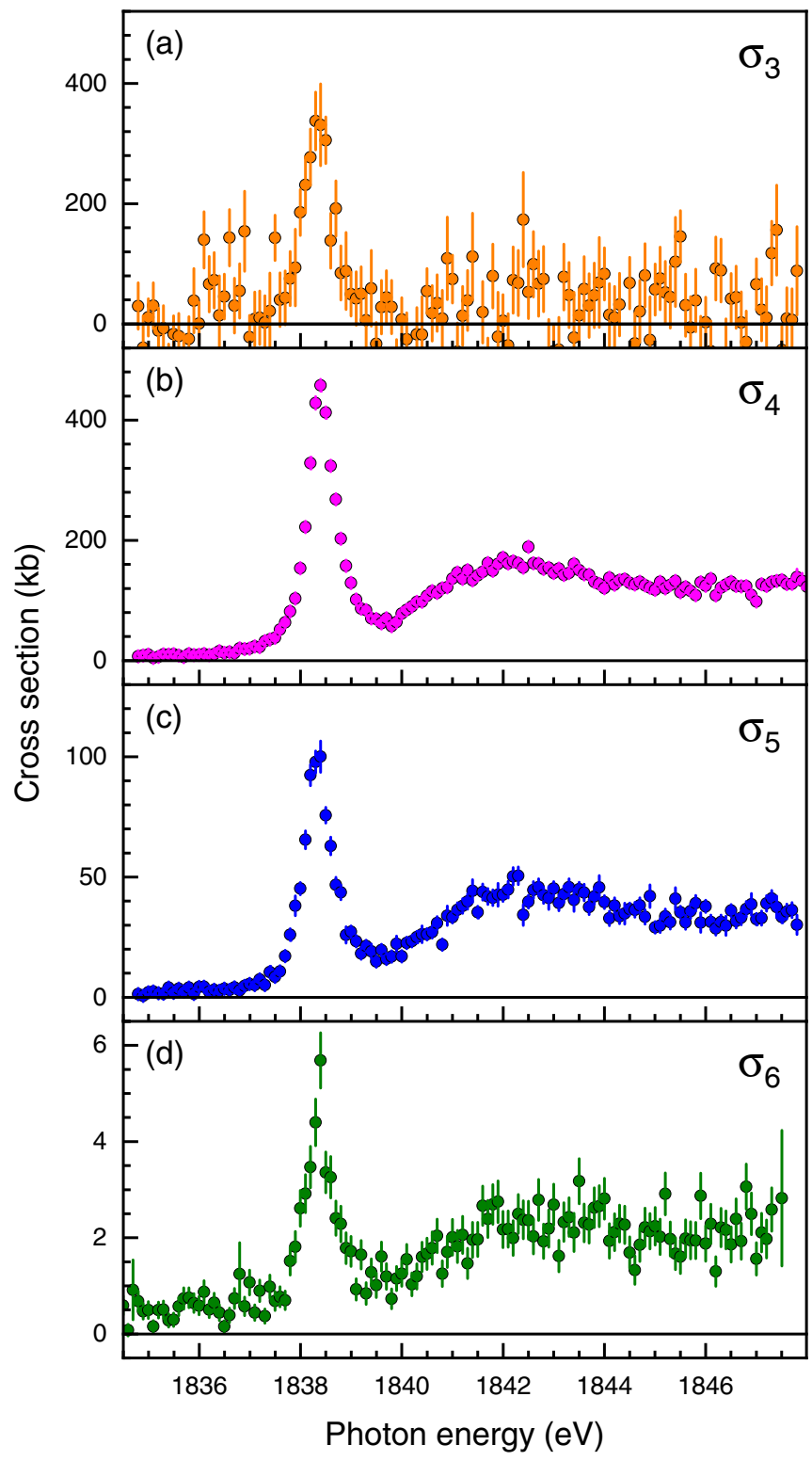

FIG. 2. High-resolution cross sections $\sigma_{m}$ for $m$-fold ( $\left.m=3-6\right)$ photodetachment of $\mathrm{Si}^{-}$ions in the energy region of the nearthreshold resonances. The width of the monochromator exit slit was $50 \mu \mathrm{m}$.

from some obviously not quite correct relative line strengths, the general agreement between experiment and theory is very satisfying considering the complexity of the problem under study. Furthermore, we point out that, at energies above $\sim 1846 \mathrm{eV}$, our theoretical cross section for photoabsorption of $\mathrm{Si}^{-}$agrees excellently with the absorption cross section for photoabsorption of neutral silicon [34]. This supports our earlier assumption that the presence of the extra electron in $\mathrm{Si}^{-}$does not influence the cross section for direct inner-shell detachment.

In Fig. 4(a), the outcome of our cascade calculations, i.e., the distribution of product-ion charge states $q=m-1$ resulting from $m$-fold photodetachment (stars in Fig. 4), is compared to the corresponding experimental values

$$
f_{q}=\sigma_{q+1} / \sigma_{\Sigma}
$$




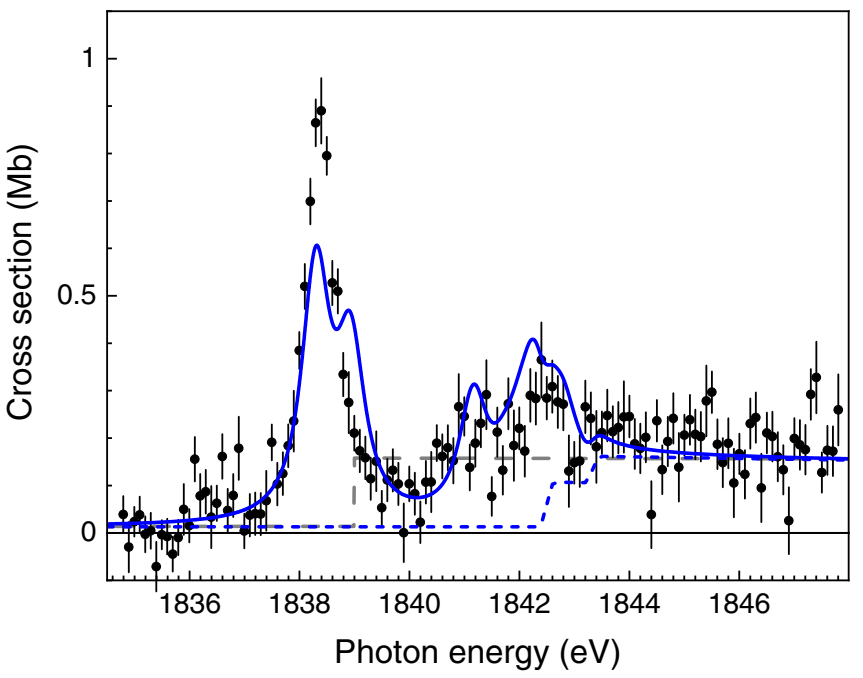

FIG. 3. Comparison between the high-resolution experimental sum cross section $\sigma_{\Sigma}$ (sum of the cross sections displayed in Fig. 2) with the present theoretical absorption cross section (full line). The short-dashed line represents the contribution by direct detachment of a single $L$-shell or $K$-shell electron to the absorption cross section. As in Fig. 1, the long-dashed curve is the cross section for photoabsorption of neutral silicon atoms [34].

The behavior of these charge-state fractions can be qualitatively understood as follows. The cross sections below $\sim 1837 \mathrm{eV}$, i.e., below the first $K$-shell excitation energy are mainly due to $L$-shell detachment and the subsequent autoionization that fills the $L$-shell hole. Over the narrow displayed energy range below $1837 \mathrm{eV}$, the branching ratios for the production of the various final charge states are virtually constant. Therefore, all partial cross sections $\sigma_{q+1}$ have a similar progression as functions of photon energy and, thus, also the fractions $f_{q}$. This changes when a $K$-shell hole is created by excitation or direct detachment. The subsequent Auger

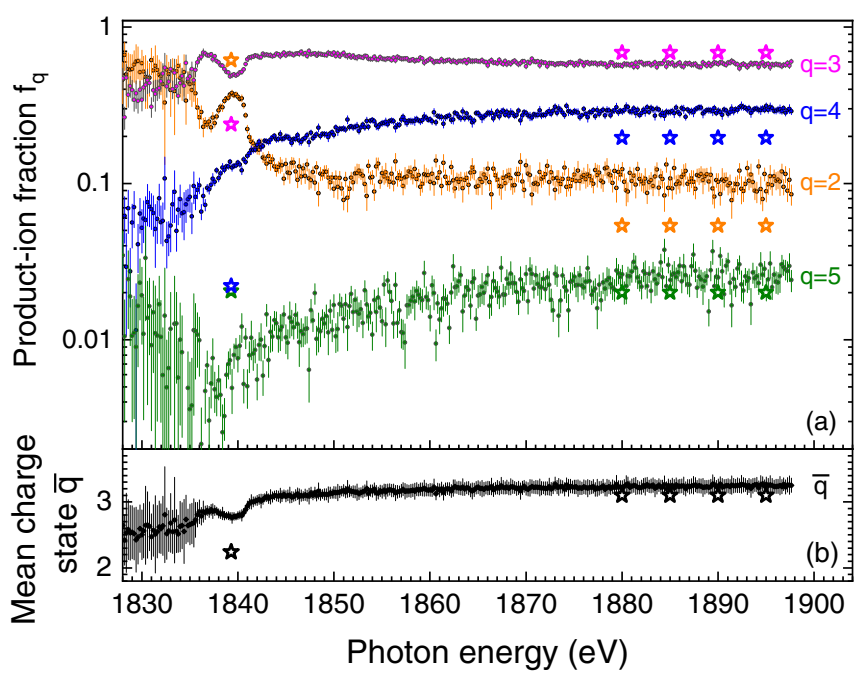

FIG. 4. Experimental (small filled circles with error bars) and theoretical (large stars) $\mathrm{Si}^{q+}$ product-ion fractions [Eq. (3), panel (a)] and mean charge state $\bar{q}=\sum_{q} q f_{q}$ [panel (b)]. processes that fill the $K$-shell hole significantly influence the branching ratios giving higher weights to the higher charge states. This is apparent for the $1 s \rightarrow 3 p$ resonance at about $1838 \mathrm{eV}$ where the fraction $f_{3}$ rises to above the fraction $f_{2}$. A similar behavior also occurs (less drastically) at the $K$-shell detachment-threshold energy of $\sim 1841 \mathrm{eV}$.

At energies above $\sim 1880 \mathrm{eV}$, the distribution results almost exclusively from direct detachment of a $K$-shell electron and the subsequent Auger cascade that produces the various product-ion charge states. The calculated fractions are of the correct orders of magnitude. For individual charge states the theoretical fractions deviate from the corresponding experimental values by factors of up to 2 . However, these uncertainties average out when the mean product-ion charge state is considered [Fig. 4(b)].

We attribute the discrepancies between the experimental and theoretical $f_{g}$ values to the approximations that we had to apply for making the cascade computations tractable (see also Sec. II). The product-ion distributions in our computations are quite strongly affected by the thresholds for the subsequent Auger processes. Because we could only accommodate an averaged single-configuration approach, the fine structure is poorly taken into account and does not describe all the ionization channels properly. Very likely, this is the main reason that the theoretical predictions for $f_{2}$ and $f_{4}$ are underrated. In the future, we hope to advance our cascade simulations, although this will require some major developments and tests for some simpler system with, say, $Z<10$. In the present cascade model, the ion fractions $f_{0}$ and $f_{1}$ are very tiny and cannot be compared directly with the experimental data. Moreover, the current cascade model does not predict any ions in the $q \geqslant 6$ channels. More accurate predictions for the higher product-ion charge states will also require the incorporation of simultaneous double-detachment processes, either during the initial detachment and/or the subsequent decay (see, e.g., Refs. [36,37]).

Below the threshold for $K$-shell detachment, the distribution of product charge states depends on the individual $K$-shell excited core-hole levels. Computationally, this is even more demanding than the calculation of the cascades that follow the direct $K$-shell detachment, since the coreexcited configurations then contain one more electron as compared to the core-ionized configurations. We have performed one such calculation for the $1 s^{-1} 3 p^{4}$ configuration. The resulting product-ion fractions (stars at $\sim 1839 \mathrm{eV}$ in Fig. 4) compare less well with the experimental ones. The fractions $f_{3}$ and $f_{4}$ are underestimated by more than a factor of 2 resulting in a significantly too small mean charge state.

\section{SUMMARY AND CONCLUSIONS}

The present joint experimental and theoretical study of multiple photodetachment of the silicon anion via $K$-shell excitation and ionization extends our previous work with light ions (see Ref. [7] and references therein) to an atomic system with a partly occupied $M$ shell. This additional valence shell challenges experiment and theory alike. The experimental challenge arises from the fact that $K$-shell detachment cross sections decrease with increasing nuclear charge. The cross 
section for the direct detachment of a $1 \mathrm{~s}$ electron is an order of magnitude smaller for $\mathrm{Si}^{-}$as compared to $\mathrm{C}^{-}$. The photonion merged-beams method as implemented at the PETRA III synchrotron successfully proved capable of meeting this challenge, with the high photon flux from the XUV beamline P04 and the high product selectivity of the PIPE setup being the key assets [23].

The present theoretical calculations were mainly challenged by the strong correlation effects that govern all anions and by the complexity of the deexcitation cascades following after the initial creation of a $K$-shell vacancy. In particular, the presence of the $M$-shell places additional demands on the cascade calculations as compared to our earlier studies with light negative ions that have been summarized in Ref. [7]. The large amount of levels and transitions to be followed in such calculations requires a systematic and consistent approach as now offered by the recently developed JAC toolbox $[17,18]$.

The measured cross sections for net triple to sixfold photodetachment exhibit a threshold for direct photodetachment of one $K$-shell electron. This threshold is masked by resonances associated with $1 s \rightarrow 3 p$ and $1 s \rightarrow 4 p$ excitations in the language of single-particle excitations. The calculated resonance positions agree with the values from a high-resolution measurement by less than $2 \mathrm{eV}$. While the calculated relative resonance strengths agree reasonably well with our measurements, they leave room for improved theoretical approaches. The same is true for the fractions of the product ions as obtained from the cascade computations. These turn out to be more reliable for the deexcitation cascade that follows direct $K$-shell detachment than for the one that evolves after $1 s \rightarrow 3 p$ excitation.
The statistical quality of the present data is somewhat lower as compared to a recently published and similarly comprehensive study on multiple photodetachment of $\mathrm{C}^{-}$ions [13]. This is partly due to the already mentioned reduced cross section but also due to a lower photon flux which varies with photon energy such that it rapidly decreases at energies above $1000 \mathrm{eV}$. Since $\mathrm{C}^{-}\left([\mathrm{He}] 2 s^{2} 2 p^{3}\right)$ and $\mathrm{Si}^{-}\left([\mathrm{Ne}] 3 s^{2} 3 p^{3}\right)$ have analog open-shell structures, one might expect similarities in the progression of the detachment cross sections. Indeed, the double-resonance structure at the $K$-shell detachment threshold is common to both species. However, additional small resonances at higher energies, that were discovered in the $\mathrm{C}^{-}$triple-detachment channel, could not be detected in any of the measured $\mathrm{Si}^{-}$photodetachment channels within the present level of statistical uncertainty. Finally, we would like to mention that the experimentally derived and calculated cross sections for $\mathrm{Si}^{-}$photoabsorption might be useful for identifying silicon anions in cold cosmic gas clouds by their characteristic $\mathrm{x}$-ray absorption pattern.

\section{ACKNOWLEDGMENTS}

We acknowledge DESY (Hamburg, Germany), a member of the Helmholtz Association HGF, for the provision of experimental facilities. Parts of this research were carried out at PETRA III and we would like to thank Kai Bagschik, Frank Scholz, Jörn Seltmann, and Moritz Hoesch for assistance in using beamline P04. We are grateful for support from Bundesministerium für Bildung und Forschung within the "Verbundforschung" funding scheme (Grants No. 05K19GU3 and No. 05K19RG3) and from Deutsche Forschungsgemeinschaft (DFG, Project No. 389115454).
[1] D. Leimbach, J. Karls, Y. Guo, R. Ahmed, J. Ballof, L. Bengtsson, F. Boix Pamies, A. Borschevsky, K. Chrysalidis, E. Eliav, D. Fedorov, V. Fedosseev, O. Forstner, N. Galland, R. F. Garcia Ruiz, C. Granados, R. Heinke, K. Johnston, A. Koszorus, U. Köster, M. K. Kristiansson, Y. Liu, B. Marsh, P. Molkanov, L. F. Pašteka, J. P. Ramos, E. Renault, M. Reponen, A. Ringvall-Moberg, R. E. Rossel, D. Studer, A. Vernon, J. Warbinek, J. Welander, K. Wendt, S. Wilkins, D. Hanstorp, and S. Rothe, The electron affinity of astatine, Nat. Commun. 11, 3824 (2020).

[2] M. S. Safronova, C. Cheung, M. G. Kozlov, S. E. Spielman, N. D. Gibson, and C. W. Walter, Predicting quasibound states of negative ions: $\mathrm{La}^{-}$as a test case, Phys. Rev. A 103, 022819 (2021).

[3] T. W. Gorczyca, Inner-shell photodetachment dynamics, Radiat. Phys. Chem. 70, 407 (2004).

[4] V. K. Ivanov, Theoretical studies of photodetachment, Radiat. Phys. Chem. 70, 345 (2004).

[5] H. Kjeldsen, Photoionization cross sections of atomic ions from merged-beam experiments, J. Phys. B 39, R325 (2006).

[6] R. C. Bilodeau, N. D. Gibson, C. W. Walter, A. Aguilar, and N. Berrah, Inner-shell photodetachment: Shape and Feshbach resonances of anions, J. Electron Spectrosc. Relat. Phenom. 185, 219 (2012).
[7] S. Schippers, A. Perry-Sassmannshausen, T. Buhr, M. Martins, S. Fritzsche, and A. Müller, Multiple photodetachment of atomic anions via single and double core-hole creation, J. Phys. B 53, 192001 (2020).

[8] H. Kjeldsen, P. Andersen, F. Folkmann, B. Kristensen, and T. Andersen, Inner-shell photodetachment of $\mathrm{Li}^{-}$, J. Phys. B 34, L353 (2001).

[9] N. Berrah, J. D. Bozek, A. A. Wills, G. Turri, H.-L. Zhou, S. T. Manson, G. Akerman, B. Rude, N. D. Gibson, C. W. Walter, L. VoKy, A. Hibbert, and S. M. Ferguson, K-Shell Photodetachment of $\mathrm{Li}^{-}$: Experiment and Theory, Phys. Rev. Lett. 87, 253002 (2001).

[10] N. Berrah, R. C. Bilodeau, I. Dumitriu, J. D. Bozek, N. D. Gibson, C. W. Walter, G. D. Akerman, O. Zatsarinny, and T. W. Gorczyca, Shape resonances in the absolute $K$-shell photodetachment of B ${ }^{-}$, Phys. Rev. A 76, 032713 (2007).

[11] N. D. Gibson, C. W. Walter, O. Zatsarinny, T. W. Gorczyca, G. D. Ackerman, J. D. Bozek, M. Martins, B. M. McLaughlin, and $\mathrm{N}$. Berrah, $K$-shell photodetachment from $\mathrm{C}^{-}$: Experiment and theory, Phys. Rev. A 67, 030703(R) (2003).

[12] C. W. Walter, N. D. Gibson, R. C. Bilodeau, N. Berrah, J. D. Bozek, G. D. Ackerman, and A. Aguilar, Shape resonance in $K$-shell photodetachment from $\mathrm{C}^{-}$, Phys. Rev. A 73, 062702 (2006). 
[13] A. Perry-Sassmannshausen, T. Buhr, A. Borovik, Jr., M. Martins, S. Reinwardt, S. Ricz, S. O. Stock, F. Trinter, A. Müller, S. Fritzsche, and S. Schippers, Multiple Photodetachment of Carbon Anions Via Single and Double Core-Hole Creation, Phys. Rev. Lett. 124, 083203 (2020).

[14] N. D. Gibson, R. C. Bilodeau, C. W. Walter, D. Hanstorp, A. Aguilar, N. Berrah, D. J. Matyas, Y.-G. Li, R. M. Alton, and S. E. Lou, K-shell photodetachment from $\mathrm{O}^{-}$, J. Phys.: Conf. Ser. 388, 022102 (2012).

[15] S. Schippers, R. Beerwerth, L. Abrok, S. Bari, T. Buhr, M. Martins, S. Ricz, J. Viefhaus, S. Fritzsche, and A. Müller, Prominent role of multielectron processes in $K$-shell double and triple photodetachment of oxygen anions, Phys. Rev. A 94, 041401(R) (2016).

[16] A. Müller, A. Borovik, Jr., S. Bari, T. Buhr, K. Holste, M. Martins, A. Perry-Saßmannshausen, R. A. Phaneuf, S. Reinwardt, S. Ricz, K. Schubert, and S. Schippers, Near-KEdge Double and Triple Detachment of the $\mathrm{F}^{-}$Negative Ion: Observation of Direct Two-Electron Ejection by a Single Photon, Phys. Rev. Lett. 120, 133202 (2018).

[17] S. Fritzsche, A fresh computational approach to atomic structures, processes and cascades, Comput. Phys. Commun. 240, 1 (2019).

[18] S. Fritzsche, P. Palmeri, and S. Schippers, Atomic cascade computations, Symmetry 13, 520 (2021).

[19] T. J. Millar, C. Walsh, and T. A. Field, Negative ions in space, Chem. Rev. 117, 1765 (2017).

[20] E. Gatuzz, T. W. Gorczyca, M. F. Hasoglu, N. S. Schulz, L. Corrales, and C. Mendoza, Silicon ISM x-ray absorption: The gaseous component, Mon. Not. R. Astron. Soc. 498, L20 (2020).

[21] S. Schippers, A. L. D. Kilcoyne, R. A. Phaneuf, and A. Müller, Photoionisation of ions with synchrotron radiation: From ions in space to atoms in cages, Contemp. Phys. 57, 215 (2016).

[22] S. Schippers, S. Ricz, T. Buhr, A. Borovik Jr., J. Hellhund, K. Holste, K. Huber, H.-J. Schäfer, D. Schury, S. Klumpp, K. Mertens, M. Martins, R. Flesch, G. Ulrich, E. Rühl, T. Jahnke, J. Lower, D. Metz, L. P. H. Schmidt, M. Schöffler, J. B. Williams, L. Glaser, F. Scholz, J. Seltmann, J. Viefhaus, A. Dorn, A. Wolf, J. Ullrich, and A. Müller, Absolute cross sections for photoionization of $\mathrm{Xe}^{q+}$ ions $(1 \leqslant q \leqslant 5)$ at the $3 \mathrm{~d}$ ionization threshold, J. Phys. B 47, 115602 (2014).

[23] S. Schippers, T. Buhr, A. Borovik Jr., K. Holste, A. PerrySassmannshausen, K. Mertens, S. Reinwardt, M. Martins, S. Klumpp, K. Schubert, S. Bari, R. Beerwerth, S. Fritzsche, S. Ricz, J. Hellhund, and A. Müller, The photon-ion mergedbeams experiment PIPE at PETRA III: The first five years, X-Ray Spectrom. 49, 11 (2020).

[24] J. Viefhaus, F. Scholz, S. Deinert, L. Glaser, M. Ilchen, J. Seltmann, P. Walter, and F. Siewert, The variable polarization XUV beamline P04 at PETRA III: Optics, mechanics and their performance, Nucl. Instrum. Methods A 710, 151 (2013).
[25] R. Middleton, A versatile high intensity negative ion source, Nucl. Instrum. Methods 220, 105 (1984).

[26] D. Müll, F. Grussie, K. Blaum, S. George, J. Göck, M. Grieser, R. von Hahn, Z. Harman, A. Kálosi, C. H. Keitel, C. Krantz, C. Lyu, O. Novotný, F. Nuesslein, D. Paul, V. C. Schmidt, S. Singh, S. Sunil Kumar, X. Urbain, A. Wolf, and H. Kreckel, Metastable states of $\mathrm{Si}^{-}$observed in a cryogenic storage ring, Phys. Rev. A 104, 032811 (2021).

[27] M. Scheer, R. C. Bilodeau, C. A. Brodie, and H. K. Haugen, Systematic study of the stable states of $\mathrm{C}^{-}, \mathrm{Si}^{-}, \mathrm{Ge}^{-}$, and $\mathrm{Sn}^{-}$via infrared laser spectroscopy, Phys. Rev. A 58, 2844 (1998).

[28] K. Rinn, A. Müller, H. Eichenauer, and E. Salzborn, Development of single-particle detectors for keV ions, Rev. Sci. Instrum. 53, 829 (1982).

[29] M. Kato, Y. Morishita, M. Oura, H. Yamaoka, Y. Tamenori, K. Okada, T. Matsudo, T. Gejo, I. H. Suzuki, and N. Saito, Absolute photoionization cross sections with ultra-high energy resolution for $\mathrm{Ar}, \mathrm{Kr}, \mathrm{Xe}$ and $\mathrm{N}_{2}$ in inner-shell ionization regions, J. Electron Spectrosc. Relat. Phenom. 160, 39 (2007).

[30] A. Müller, D. Bernhardt, A. Borovik Jr., T. Buhr, J. Hellhund, K. Holste, A. L. D. Kilcoyne, S. Klumpp, M. Martins, S. Ricz, J. Seltmann, J. Viefhaus, and S. Schippers, Photoionization of $\mathrm{Ne}$ atoms and $\mathrm{Ne}^{+}$ions near the $\mathrm{K}$ edge: Precision spectroscopy and absolute cross-sections, Astrophys. J. 836, 166 (2017).

[31] A. Müller, E. Lindroth, S. Bari, A. Borovik Jr., P.-M. Hillenbrand, K. Holste, P. Indelicato, A. L. D. Kilcoyne, S. Klumpp, M. Martins, J. Viefhaus, P. Wilhelm, and S. Schippers, Photoionization of metastable heliumlike $\mathrm{C}^{4+}\left(1 s 2 s^{3} S_{1}\right)$ ions: Precision study of intermediate doubly excited states, Phys. Rev. A 98, 033416 (2018).

[32] S. Fritzsche, JAC: User Guide, Compendium \& Theoretical Background, https://github.com/OpenJAC/JAC.jl.

[33] S. Fritzsche, B. Fricke, and W.-D. Sepp, Reduced $L_{1}$ level width and Coster-Kronig yields by relaxation and continuum interactions in atomic zinc, Phys. Rev. A 45, 1465 (1992).

[34] B. L. Henke, E. M. Gullikson, and J. C. Davis, X-ray interactions: Photoabsorption, scattering, transmission, and reflection at $\mathrm{E}=50-30,000 \mathrm{eV}, \mathrm{Z}=1-92$, At. Data Nucl. Data Tables 54, 181 (1993).

[35] J. L. Campbell and T. Papp, Widths of the atomic K-N7 levels, At. Data Nucl. Data Tables 77, 1 (2001).

[36] R. Beerwerth, T. Buhr, A. Perry-Sassmannshausen, S. O. Stock, S. Bari, K. Holste, A. L. D. Kilcoyne, S. Reinwardt, S. Ricz, D. W. Savin, K. Schubert, M. Martins, A. Müller, S. Fritzsche, and S. Schippers, Near L-edge single and multiple photoionization of triply charged iron ions, Astrophys. J. 887, 189 (2019).

[37] A. Müller, M. Martins, A. Borovik, T. Buhr, A. PerrySassmannshausen, S. Reinwardt, F. Trinter, S. Schippers, S. Fritzsche, and A. S. Kheifets, Role of $L$-shell single and double core-hole production and decay in $m$-fold $(1 \leqslant m \leqslant 6)$ photoionization of the $\mathrm{Ar}^{+}$ion, Phys. Rev. A 104, 033105 (2021). 